\title{
Privacy Preserving User Authentication Scheme based on Password and Smart Card for Multi-Server Environment
}

\section{Stanley Mlatho}

University of Malawi

\section{Prince Chirwa}

University of Malawi

Yesaya Gabriel

University of Malawi

Hyunsung Kim ( $\nabla$ kim@kiu.ac.kr)

Kyungil University https://orcid.org/0000-0002-7814-7454

\section{Research}

Keywords: User authentication, Multi-server, Smart card, Privacy, ProVerif, BAN logic

Posted Date: June 15th, 2020

DOI: https://doi.org/10.21203/rs.3.rs-35155/v1

License: (c) (i) This work is licensed under a Creative Commons Attribution 4.0 International License. Read Full License 


\section{Abstract}

Advancement in information and communication technology provides a scalable platform for various communication services, where a remote user can access the server from anywhere and anytime. The multi-server environment introduces a scalable platform such that a user can interact with any server using single registration. These services adopt authentication schemes in order to ensure secure and privacy preserving access to the resources. Recently Zhao et al. proposed a user authentication scheme based on password and smart card for the multi-server environment. They argued that their scheme is secure from well-known attacks. In this paper, we first identify that Zhao et al.'s scheme suffers from the denial of service attack and the privacy attack and does not provide user friendliness. After that, we propose a new privacy preserving user authentication scheme as a remedy scheme of Zhao et al.'s scheme. Through the rigorous formal and informal security analysis, we show that our scheme is secure against various known attacks including attacks founded in Zhao et al.'s scheme. Furthermore, we simulate our scheme for the formal security verification using ProVerif tool.

\section{Full Text}

This preprint is available for download as a PDF.

\section{Figures}




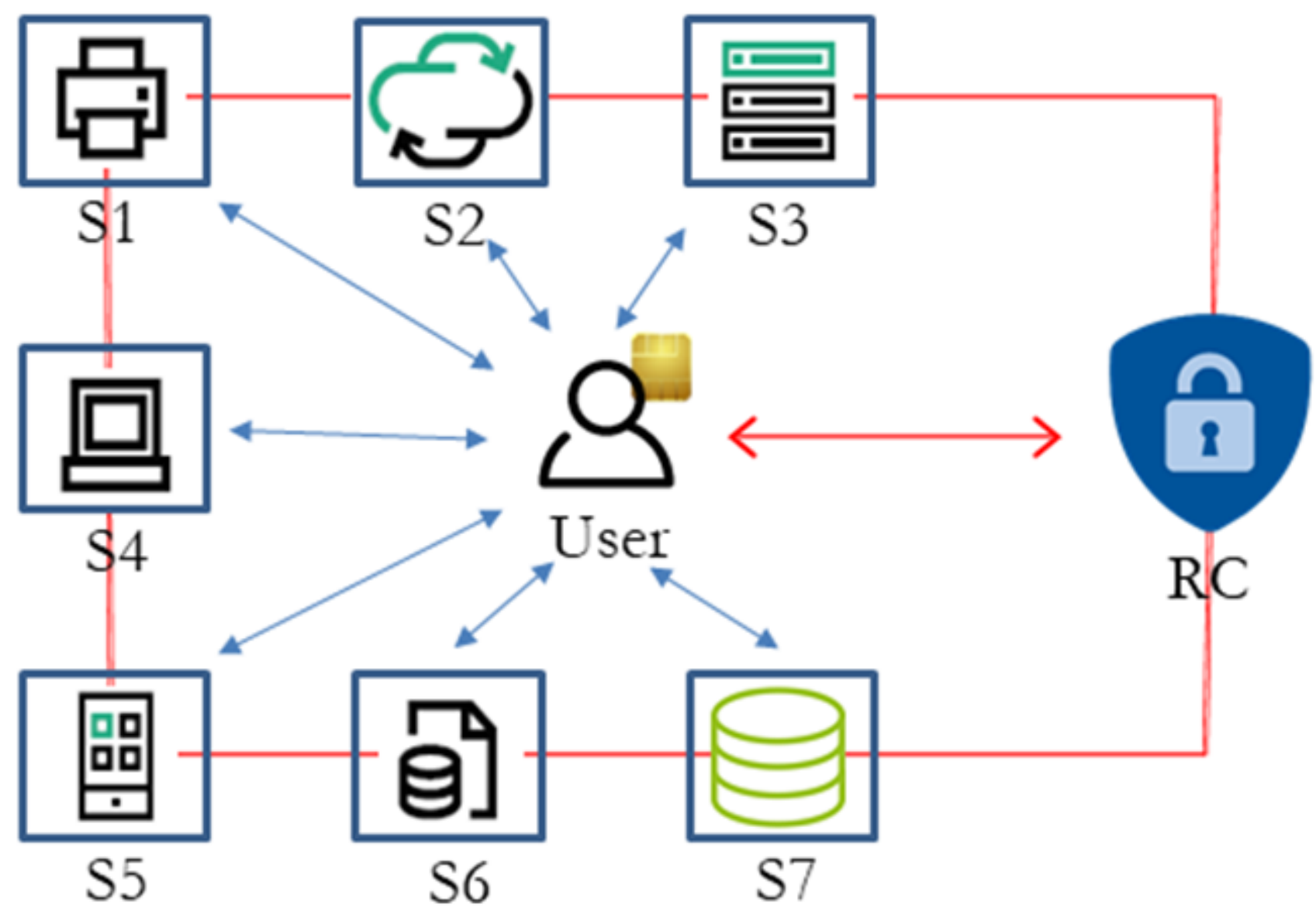

Figure 1

System environment. 
Service provider $S_{j}$

Selects $S I D$

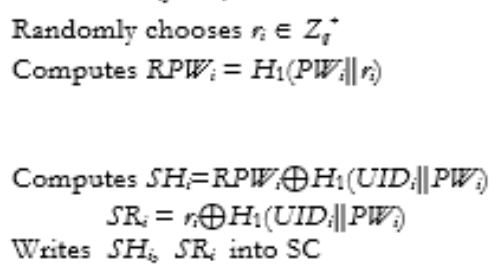

(a) Server registration phase.

User $U_{i}$

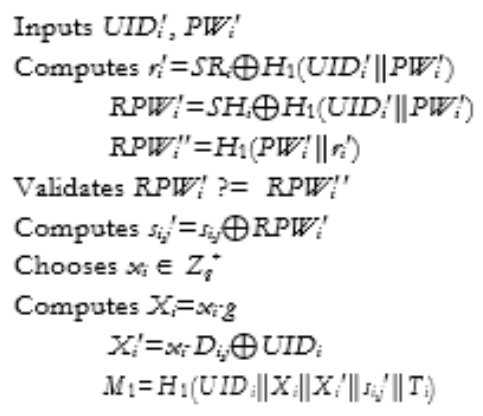

(b) User registration phase.

$$
\text { Registration center } R C
$$

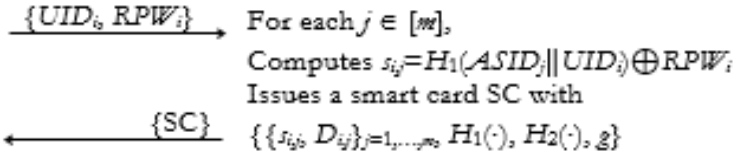

Service provider $S_{j}$

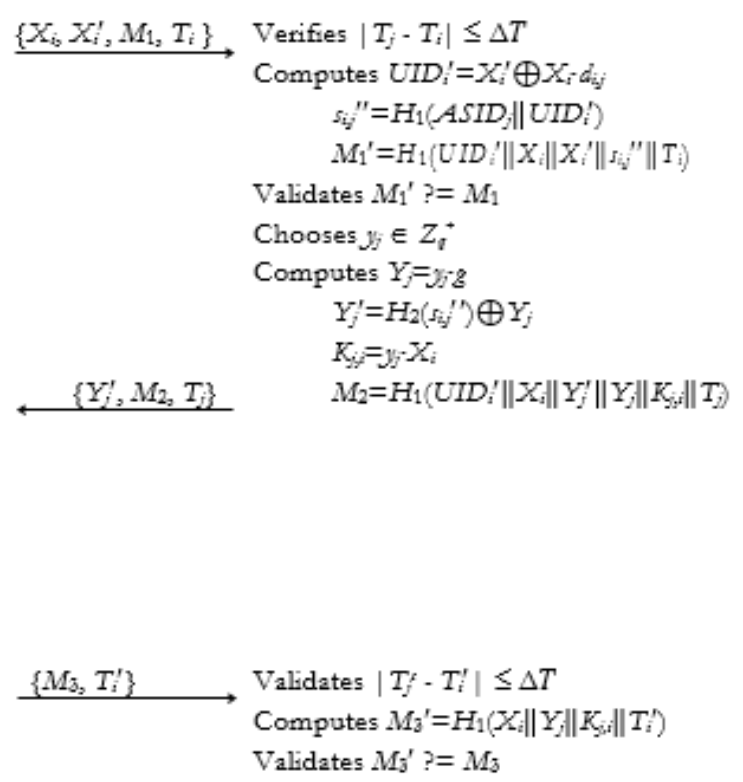

(c) Authentication phase.

\section{Figure 2}

Proposed privacy preserving user authentication scheme. 


\section{ProVerif text output:}

Starting query jai-event (Slend $(t))=\Rightarrow$ jai-event (Slbegin $(t))$

RESULT jai-event (Sllend $(t))=\Rightarrow$ jai-event (SUlbegin( $(t)$ ) is true.

-- Query not attacker(svalued []); not attacker(svalueb[]) in process 0

Completing...

200 rules inserted. The rule base contains 191 rules. 17 rules in the queve.

Starting query not attacker (svalued [])

RESULT not attacker(svalued []) is true.

Starting query not at tacker (svalueb[])

RESULT not attacker(sualueB[1]) is true.

Verification summary:

Query iai-event (USend $(t))=\Rightarrow$ iai-event (USbegin(t)) is true.

Query iai-event (Sllend $(t))=\Rightarrow$ iai-event (SUbegin(t)) is true.

Query not attacker(svalues[1]) is true.

Query not attacker(svalueB[]) is true.

Figure 3

The results of ProVerif validation.

\section{Supplementary Files}

This is a list of supplementary files associated with this preprint. Click to download.

- authorbiography.doc

- proverif.pv 